\title{
A Suggestion Complementing the Magic Numbers Interpretation of the Nuclear Fission Phenomena
}

\author{
Faustino Menegus \\ F. Menegus V. Europa, Bussero, Italy \\ Email: menegus.faustino@gmail.com
}

How to cite this paper: Menegus, F. (2018) A Suggestion Complementing the Magic Numbers Interpretation of the $\mathrm{Nu}-$ clear Fission Phenomena. World Journal of Nuclear Science and Technology, 8, 11-22. https://doi.org/10.4236/wjnst.2018.81002

Received: November 21, 2017

Accepted: January 23, 2018

Published: January 26, 2018

Copyright $\odot 2018$ by author and Scientific Research Publishing Inc. This work is licensed under the Creative Commons Attribution International License (CC BY 4.0).

http://creativecommons.org/licenses/by/4.0/

\section{(c) (i) Open Access}

\begin{abstract}
Ideas, solely related on the nuclear shell model, fail to give an interpretation of the experimental central role of ${ }_{54} \mathrm{Xe}$ in the asymmetric fission of actinides. The same is true for the $\beta$-delayed fission of ${ }^{180} \mathrm{Tl}$ to ${ }^{80} \mathrm{Kr}$ and ${ }^{100} \mathrm{Ru}$. The representation of the natural isotopes, in the Z-Neutron Excess plane, suggests the importance of the of the Neutron Excess evolution mode in the fragments of the asymmetric actinide fission and in the fragments of the $\beta$-delayed fission of ${ }^{180} \mathrm{Tl}$. The evolution mode of the Neutron Excess, hinged at $\mathrm{Kr}$ and $\mathrm{Xe}$, is directed by the 50 and 82 neutron magic numbers. The present isotope representation offers a frame for the interpretation of the post fission evaporation of neutrons, higher for the $A_{L}$ compared to the $A_{H}$ fragments, a tenet in nuclear fission. Further enlightened is the functional meaning of the 50 proton magic number, marking the start of the yield rise of the $A_{H}$ fragments in actinide fission.
\end{abstract}

\section{Keywords}

Nuclear Structure, Neutron Excess, Magic Numbers, Isotons, Binding Energy, $\mathrm{Kr}, \mathrm{Xe}$, Actinides

\section{Introduction}

The strong and short-range nucleon-nucleon attractive forces, granting both the internal nuclear cohesion and the nuclear surface tension, are still at the heart of the interpretation of the atomic nuclear fission, according to the Liquid Drop Model (LDM) [1]. Yet while it is possible to explain the mass and charge symmetrical splitting with the LDM, this is not the case for the asymmetrical nuclear splitting. In this case, exemplified by the nuclear reaction ${ }^{235} \mathrm{U}_{(\mathrm{nt}, \mathrm{f})}$, heavy and 
light fragments of mass close to $140 \mathrm{and} 80 \mathrm{amu}$ are produced, bearing different charges.

The nuclear fission, discovered in 1934, was indeed an asymmetric fission. Otto Han [2] was able to reveal the presence of ${ }_{56} \mathrm{Ba}$ in the nuclear fragments, an element incompatible with the current ideas of that time and incompatible with the LDM soon developed [1]. Nuclear deformation [3] enabled nuclei to progress, under a variety of energy stimuli, towards the separation of the nuclear material into two distinct centres representing the nuclei of the nascent fragments of the nucleus undergoing fission. Sound models, for the interpretation of the asymmetric fission, appeared, after substantial improvements of the LDM [4] [5], only following the ideas of Strutinsky. He suggested to take into consideration the shell effects [6] [7] of the proton and neutron magic numbers of the nascent fragments on the Binding Energy (BE) landscape of the nuclei undergoing fission [8] [9].

As reported in the fine review of Gönnenwein [10] and in several others recent studies [11] [12] [13] [14], the asymmetric fission mode is understood to a deep level by the combination of the fundamental ideas of the LDM, the macro part of the models, to which are superimposed the shell effects of the magic numbers, the micro part of the models. An impressive work, relaying mainly on the shell features of the nascent daughter nuclei, allows a detailed description of the fission modes known as St1, St2, Superlong and Superasymmetric fission [13] [14]. Very interesting the Superlong mode substantiates the presence of the LDM physics in the asymmetric fission modes in accordance to the separation principle of the macro from the micro effects [13]. Of the asymmetric modes St1 and St2 the first-one is closer to the Superlong mode. The Superasymmetric mode instead stresses the relevance of the proton magic number 28 in the fission phenomena [15]. Unstable nuclei, typically the actinides with $Z=90$ to 100 , stimulated by thermal neutrons undergo asymmetric fission, possibly the most natural and lowest temperature nuclear fission. Very interesting these reactions where accompanied by the staggering phenomenon: the strong even-odd fluctuations of the fragments charge and mass yields of the $\mathrm{Z}$ even nuclei fission [10]. At increasing neutron energy, from 20 up to $100 \mathrm{MeV}$, the asymmetric fission replaces gradually the symmetric-one [10]. The General description of Fission Observables model, exploiting the general lows of mathematics and physics and empirical information, is presently successful in the representation of all the fission phenomena [16].

Yet important aspects of the nuclear fission are still obscure not fitting in the magic numbers interpretation of the phenomenon. The $\beta$-delayed fission of ${ }^{180} \mathrm{Tl}$ obeys the reaction, ${ }^{180} \mathrm{Hg}={ }^{80} \mathrm{Kr}+{ }^{100} \mathrm{Ru}$, instead of the reaction, ${ }^{180} \mathrm{Hg}=2{ }_{40} \mathrm{Zr}^{50}$ [17]. The last reaction is expected because of the neutron magic number 50 and of the half-magic proton number 40 of Zr. Equally the most probable nuclear splitting, with thermal neutrons or by electro-magnetic excitation, of elements from $\mathrm{Th}$ to Fm leads to an heavy fragment with an astonishing fixed charge $\mathrm{Z}=$ 
54. The light fragment instead shows an increasing $\mathrm{Z}$ value tailored to complement the charge of the starting nucleus [16]. It is suggested here that the proton numbers 36 and 54 of the elements $\mathrm{Kr}$ and $\mathrm{Xe}$, in spite of the corresponding shells absence in the shell model [6] [7], are important in the interpretation of the nuclear fission. The aim of the present paper is to provide support to this statement.

\section{Methods}

The isotopes, of any chemical element, can be defined in equivalent ways by couples of two numbers added to the element symbol: $\mathrm{Z}{ }^{\mathrm{A}} \mathrm{X} ;{ }^{\mathrm{A}} \mathrm{X}^{\mathrm{N}} ;{ }_{\mathrm{Z}} \mathrm{X}_{\mathrm{EN}} \cdot \mathrm{Z}, \mathrm{A}, \mathrm{N}$, NE, indicate the Proton, the Mass, the Neutron and the Neutron Excess numbers respectively of an isotope, with $\mathrm{NE}=\mathrm{A}-2 \mathrm{Z}$. The third mode allows the isotope representation in the $\mathrm{Z}-\mathrm{NE}$ plane. The $\mathrm{Z}, \mathrm{A}, \mathrm{N}, \mathrm{NE}$ and the isotope $\mathrm{BE}$ are derived from G. Audy et al. [18]. Note the mandatory presence, in all the three ways, of the isotope proton number either as the $\mathrm{Z}$ number or as the chemical element symbol. All the figures except numbers 6 and 7 have been developed through graphical programs (VB.net), starting from text files containing levels, properties (colors, lines dimension etc.) and all the comments to be visualized.

\section{Results}

\subsection{Isotopes Concerned in the Actinide and in the ${ }^{180} \mathrm{Tl} \beta$-Delayed Fission}

Figure 1 represents the natural isotopes of the elements from Fe to Gd that are of interest in the present work. The proton and neutron magic numbers of the shell model [6] [7] and the limits of the $\beta$-stability valley are highlighted. The salient features of the isotopic representation in the Z-NE plane, with NE $=\mathrm{A}-2 \mathrm{Z}$, have been reported [19]. Here it is sufficient to recall that the mass number of every isotope is, $A=N E+2 Z$. To pinpoint any isotope in Figure 1, its $\mathrm{NE}$ number needs to be determined; here it is subscribed behind the element symbol, as in $\mathrm{Sn}_{32}$. When appropriate are also employed the usual symbol's superscripts values of mass and neutron numbers.

The eight-nucleon isobar tracks (Figure 1) represent a further help in the determination of the isotopic mass number A [19]. Nuclei with 50 and 82 neutrons will be simply denoted 50 and 82 isotons respectively. The representation of the natural isotopes in the Z-NE plane adds together the features of the Segrè chart of nuclides with those of the Mendeleev's periodic table of the elements. A bridge between chemistry and physics.

\subsection{Binding Energy Evolution along the Isotopic Arrays with Constant NE Number}

At the monotonous progress of the $\mathrm{Z}$ number any sudden change of the $\mathrm{BE}$ of the isotopes lying on the constant NE arrays (Figure 1), will denote changes in the nuclear structure. As an example Figure 2 shows the BE evolution of the 


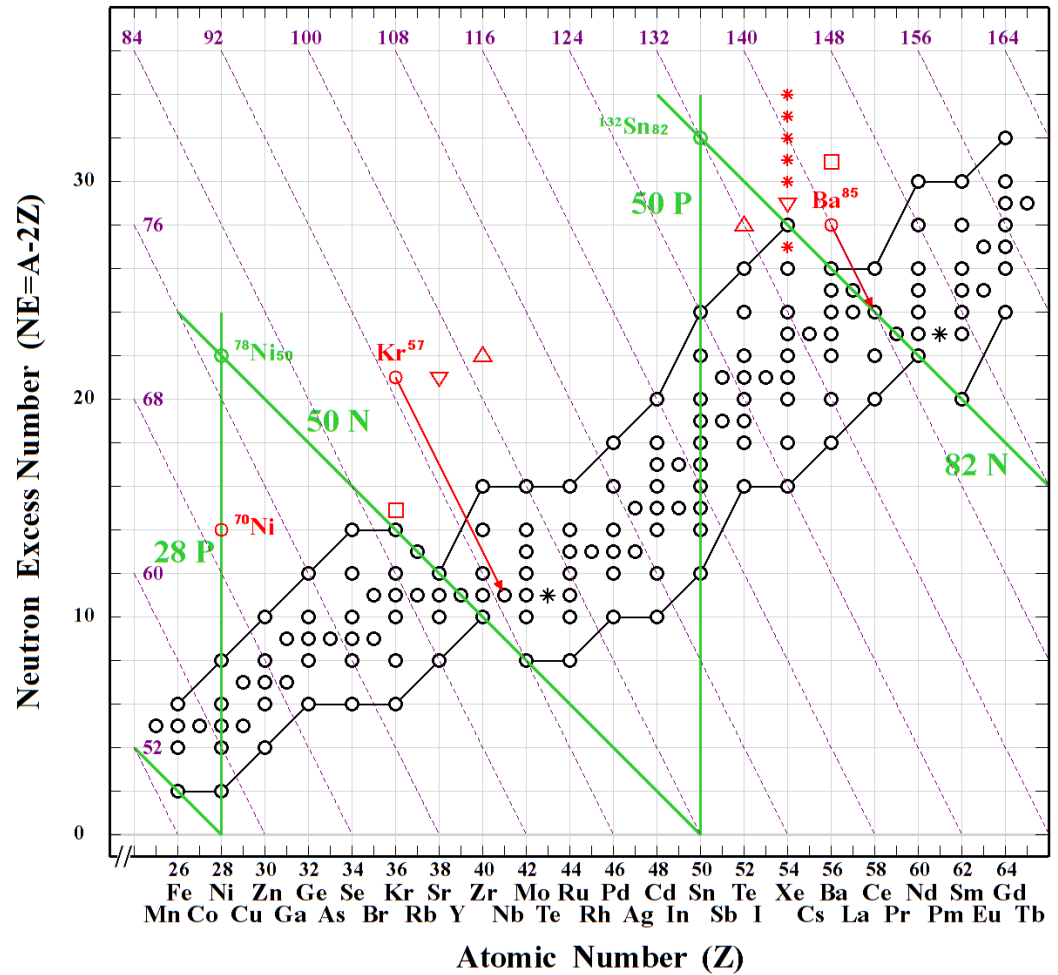

Figure 1. The representation of the natural isotopes of the elements from Fe to Gd in the Z-Ne plane [18]. Proton and neutron magic numbers and the related isoton arrays are green. The solid black lines denote the borders of the $\beta$-stability valley. Important isotopes: (a) double magic $\mathrm{Ni}$ and $\mathrm{Xe}(\mathrm{O})$; (b) ${ }^{70} \mathrm{Ni}(\mathrm{O})[15]$; (c) ${ }^{235} \mathrm{U}_{(\mathrm{n}, \mathrm{f})}$ post neutron evaporation fragments Ba-Kr and their decay to stable isotopes $(\mathrm{O} \longrightarrow$ ) [20]; (d) the three most probable pre neutron evaporation fragments couples from the electromagnetic induced fission of ${ }^{234} \mathrm{U}(\square),(\nabla),(\triangle)$ [11]; (e) the most frequent Xe fragments from actinide fission (*' [16].

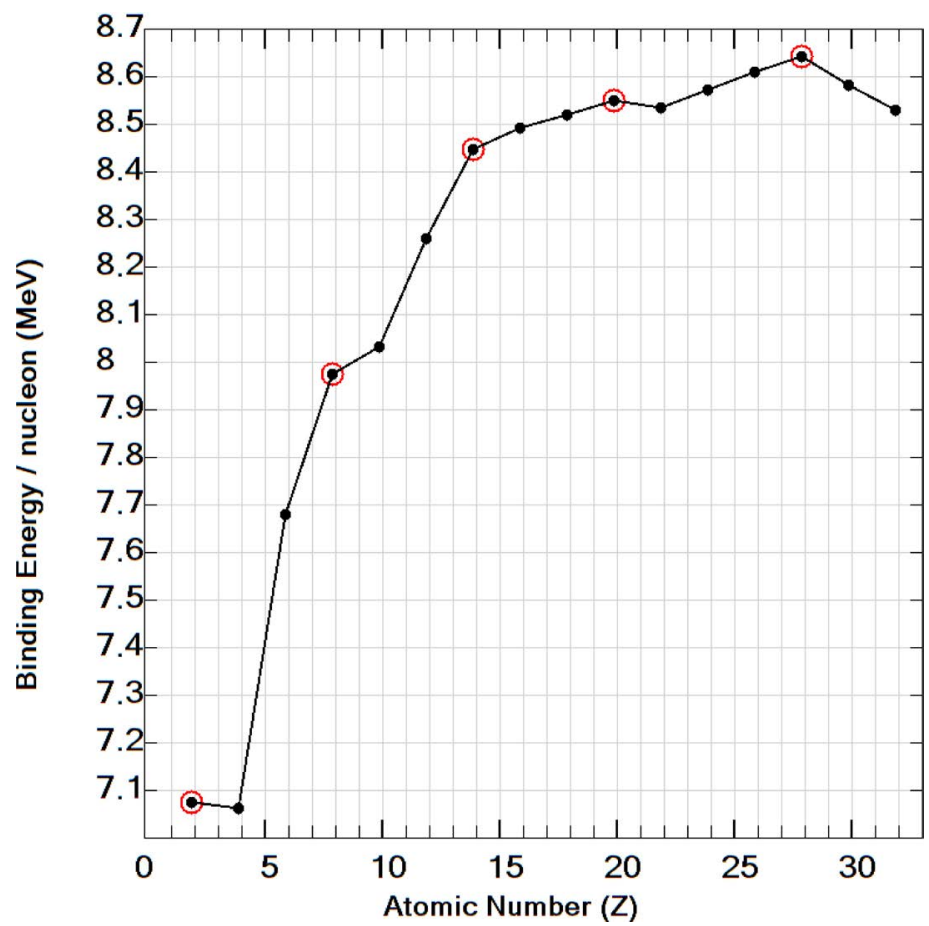

Figure 2. Evolution of the $\mathrm{BE}$ of the isotopes lying on the array with $\mathrm{EN}=0$ (Figure 1). There are marked BE changes for the double magic: ${ }^{4} \mathrm{He},{ }^{16} \mathrm{O},{ }^{40} \mathrm{Ca},{ }^{56} \mathrm{Ni}$ and for the half-magic ${ }^{14} \mathrm{~N}$. 
isotopes with zero NE, that is the special isotopes with an identical number of protons and neutrons. The double magic numbers 2, 8, 20 and 28, together with the less general half-magic $14 \mathrm{Z}$ number show sudden $\mathrm{BE}$ changes.

Figure 3 shows the $\mathrm{BE}$ evolution of the isotopes lying on the 8 to $36 \mathrm{NE}$ arrays represented in Figure 1. The $\mathrm{Z}$ and NE numbers, defining every isotope in the $\mathrm{Z}-\mathrm{NE}$ plane, allow the univocal correspondence of the Figure 3 isotopes with those of Figure 1 and vice versa. Stressed is the known relevance of the proton magic number 50. Interestingly its influence extends well beyond the limits of the $\beta$-stability valley up to $\mathrm{Sn}_{36}$. It is maximal at the double magic ${ }^{132} \mathrm{Sn}_{32}$. Also clear is the importance of the proton magic number 28. Its influence on the $\mathrm{BE}$ is marked at the upper border of the $\beta$-stability valley, shows a decrease with the $\mathrm{NE}$ increase and practically fades at the double magic ${ }^{78} \mathrm{Ni}_{22}$.

Of particular interest are the effects of the $Z$ progress on the 82 and 50 isotons: the green parabolas (Figure 3). In the first case the BE maximum is set at $Z=54$, the nuclear charge of the most probable fission mode of an extended chain of actinide nuclei [16]. In the second case the $\mathrm{BE}$ maximum is set at $\mathrm{Z}=38$, not a casual value, see discussion. The sudden $\mathrm{BE}$ changes along the NE arrays correspond to the $\mathrm{Z}$ even charge of the elements displaying the top abundance in the

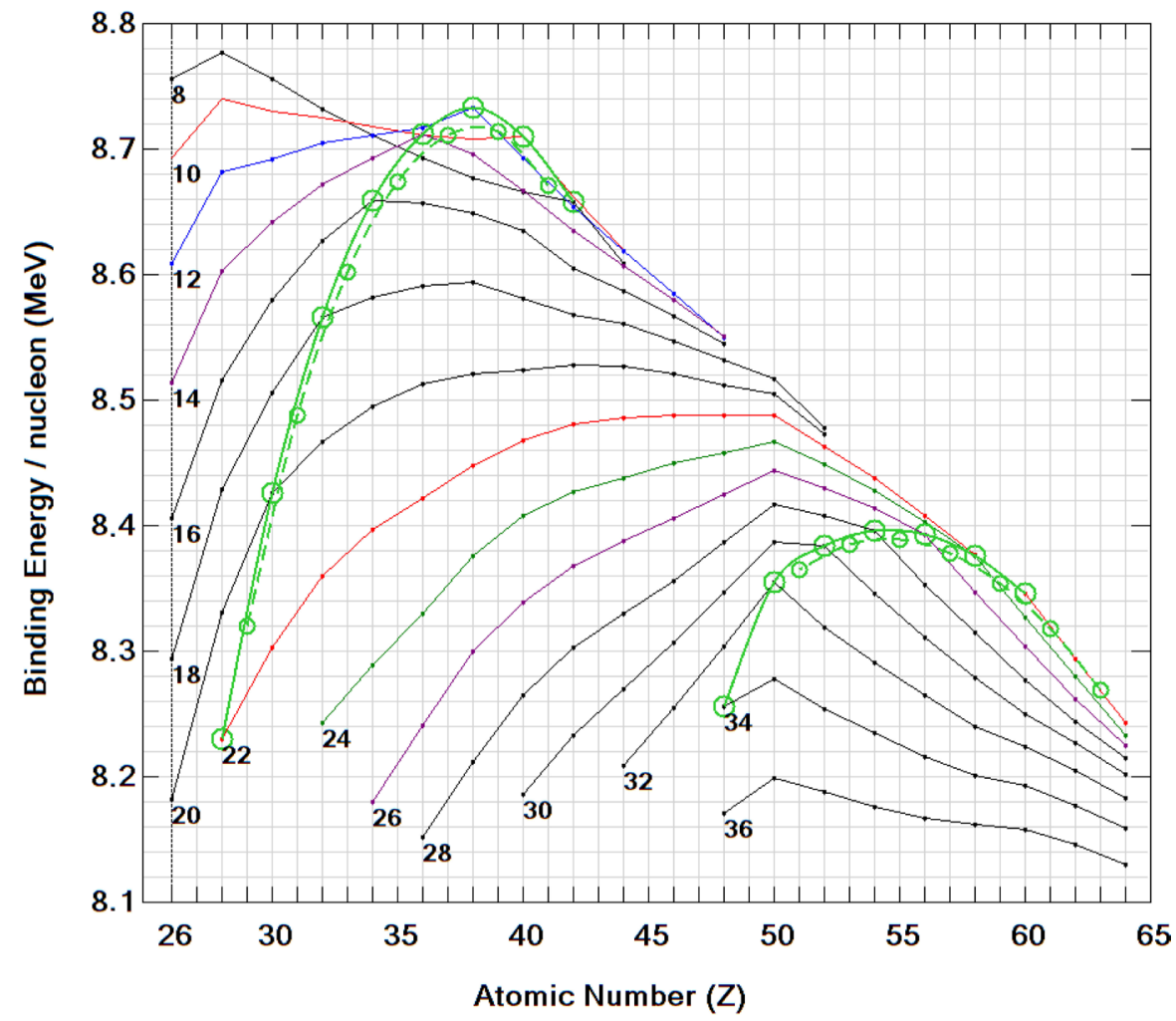

Figure 3. Evolution of the isotopic BE along the 8 to 36 even NE arrays (Figure 1) for the $\mathrm{Z}$ even elements. The prominent $\mathrm{BE}$ changes in the $\mathrm{NE}$ arrays, related to the 50 and to the 82 neutron magic numbers, are connected by a green line. Two parabolas result with maxima at ${ }_{38} \mathrm{Sr}$ and at ${ }_{54} \mathrm{Xe}$ respectively. The broken line parabolas concern odd elements and odd NE from the twin of Figure 3, not shown. The parabolas maxima shift to ${ }_{39} \mathrm{Y}$ and to ${ }_{55}$ Cs respectively. All parabola's isotopes fall onto the 50 and 82 isoton arrays (Figure 1). 


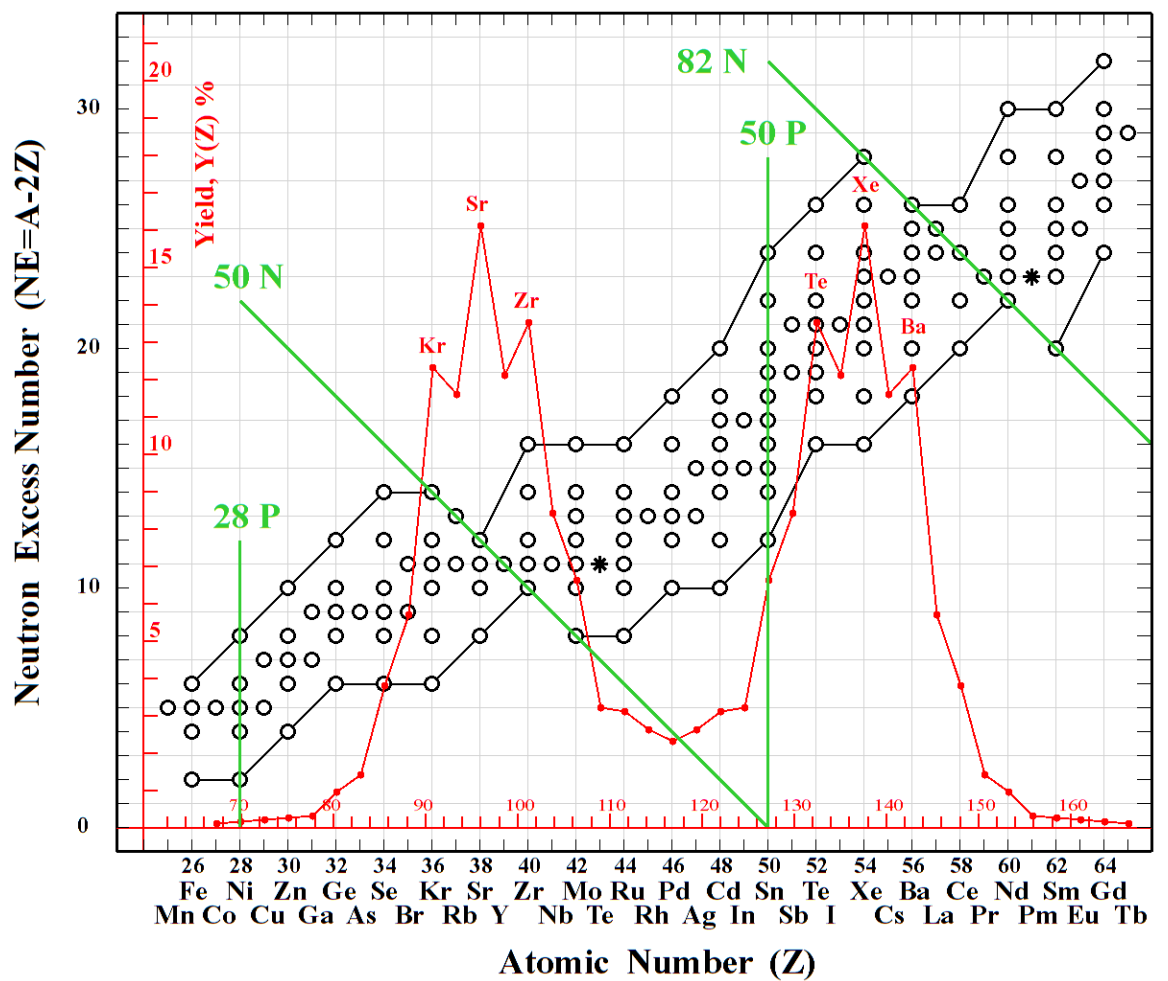

Figure 4. The electromagnetic-induced fission of ${ }^{234} \mathrm{U}$, red color, from Möller's Figure 2 [11]. The mass scale, at the top of the original figure, is here at the bottom. The Möller figure superimposes onto the isotopic layout of Figure 1. The Yeld axis refers to both the charge and mass yelds of ${ }^{234} \mathrm{U}$ fission.

charge-mass staggering typical of actinide fission (Figure 4) [11]. For the isotopes lying on the 9 to $35 \mathrm{NE}$ arrays the BE maxima of the 82 and 50 isotons shift to $Z=55$ and $Z=39$ respectively: the broken-line parabolas (Figure 3). In Figure 3, the concurrent increase of both $Z$ and NE numbers causes a fast increase of the isotopic mass numbers and the consequent fast drop in the related BE. This is why same curves are truncated and curves tend to crowd at the right lower corner.

\subsection{Relevant Results of P.Möller}

To put the results of the Figure 3 in the perspective of what learned about the nuclear fission, it is useful to reproduce (with permission) the results of the electromagnetic induced fission of ${ }^{234} \mathrm{U}$ from the Figure 2 of P. Möller [11]. It is important to keep in mind that the experimental data where converted to the massyield distribution before neutron evaporation by assuming that the proton/neutron ratio $\mathrm{Z} / \mathrm{N}$ is the same in each of the two fission fragments as in the original nucleus.

The Möller figure, placed upon the natural isotopes representation in the Z-NE plane of Figure 1, produce Figure 4.. Notably the most probable light and heavy fragments of ${ }^{234} \mathrm{U}, \mathrm{Sr}_{12}$ and $\mathrm{Xe}_{28}$ respectively, are in agreement with the maximum $\mathrm{BE}$ of these isotopes in Figure 3. The heavy fragment is an isotope of 
${ }_{54} \mathrm{Xe}$, the $\mathrm{Xe}^{82}$ isoton, the chemical element that shows the best performance in the accommodation of the EN, compared with the flanking elements (Figure 1). This is not the case for the light fragment where the choice is the ${ }_{38} \mathrm{Sr}^{50}$ isoton, in spite of the fact that the flanking element ${ }_{36} \mathrm{Kr}$ has a highest ability in the accommodation of the NE. Very important Figure 4 shows that, albeit at a low probability, the symmetric splitting is always present in the asymmetric fission mode. The magic proton number 50 marks the beginning of the mass increase for the heavy fragment (Figure 6).

\subsection{A Different View of the Figure 3 Results}

The $\mathrm{BE}$ of the 50 and 82 isotons (Figure 3 ) are compared in Figure 5, to the $\mathrm{BE}$ of the most stable natural isotope of the elements from Fe to Gd, allowing some interesting considerations. First of all the known decrease of the isotopic BE beyond $\mathrm{Fe}$ is clear. It is accompanied by a marked shoulder, with the levelling-off of the BE, between Ge and Y. A result not discussed here.

The green numbers denote the NE of the 50 and 82 isotons. The black numbers represent the NE of the most stable isotope of the elements considered. The red numbers refer to the important isotopes highlighted in Figure 1. These numbers allow an easy spotting of the corresponding isotopes both in Figure 3 and in Figure 1. It is important here to stress the different effects of the 50 and 82 neutron magic numbers on $\mathrm{Sr}, \mathrm{Kr}$ and $\mathrm{Xe}$ isotopes respectively. With $\mathrm{Sr}$ it is the neutron magic number 50 that rises the $\mathrm{BE}$ of the $\mathrm{Sr}_{12}$ isotope at the maximum causing the pick on the shoulder mentioned above. The $\mathrm{Kr}_{12}$ isotope instead is more stable than the $\mathrm{Kr}_{14}$ isotope in spite of the 50 neutrons contained in the latter isotope. Turning to the Xe isotopes, the magic neutron number $82 \mathrm{de}$ notes again the maximum $\mathrm{BE}$ of the isoton 82 family set at the $\mathrm{Xe}_{28}$ isotope. Instead the maximum $\mathrm{BE}$ of the $\mathrm{Xe}$ isotopes coincides with $\mathrm{Xe}_{18}$, clearly out of reach of the 82 neutron effects (Figure 3). Notably the 82 isoton $\mathrm{Xe}_{28}$ is the less stable of all the natural isotopes of $\mathrm{Xe}$. To summarize both $\mathrm{Kr}$ and $\mathrm{Xe}$ isotopes may reach maximum stability without the aid of the 50 and 82 neutron magic numbers. Figure 5 complements Figure 3. The important isotopes, red labelled in Figure 3, are shown in the Z-BE plane in Figure 5 together with a more extended list of the 50 and 82 isotons.

\section{Discussion}

Two principal considerations underlie the idea of the present work. The firstone concerns the display of the natural isotopes of the elements from Fe to Gd in the Z-NE plane (Figure 1). At the monotonous progress of $\mathrm{Z}$, coming near to $\mathrm{Kr}$ and $\mathrm{Xe}$, there is an increasing trend of the elements ability to harbour the NE. There is a net reduction of that ability beyond $\mathrm{Kr}$ and $\mathrm{Xe}$, and a resumption afterwards. The result are two picks in the $\mathrm{NE}$ accommodation ability at $\mathrm{Kr}$ and $\mathrm{Xe}$ characterized, in addition, by the neutron magic numbers 50 and 82 respectively. The total number of the natural isotopes per element equally shows an increase 


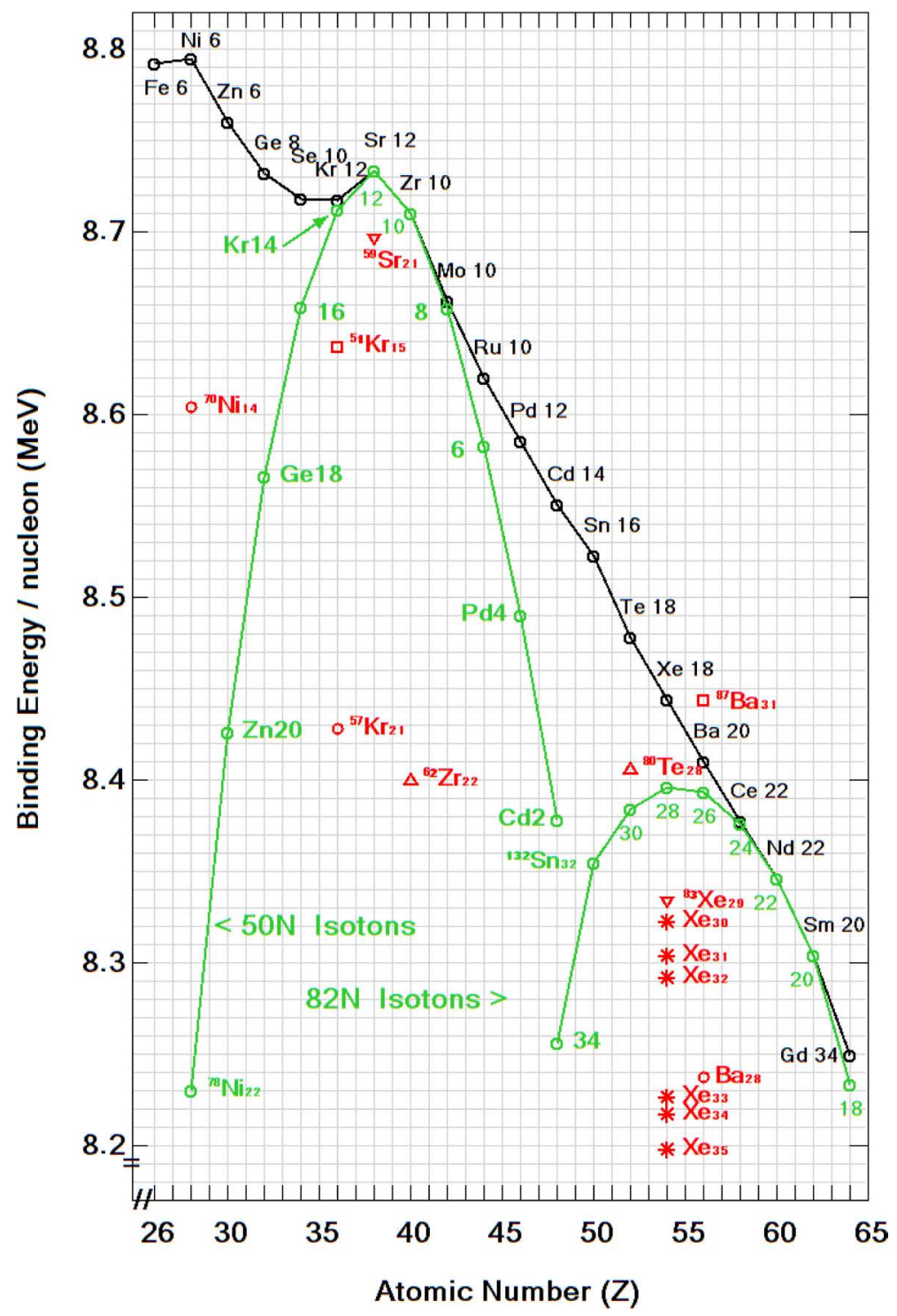

Figure 5. Comparison of the $\mathrm{BE}$ of the 50 and 82 isotons with the $\mathrm{BE}$ of the most stable isotope of the elements from Fe to Gd. At variance with Figure 3 the isoton list is more complete here. The figure also evidences the $\mathrm{BE}$ of the important isotopes highlighted in Figure 1 and legend. Note the highest BE gap between the Xeisoton and the most stable $\mathrm{Xe}$ isotope, comparted with the identical gap of $\mathrm{Kr}$ isotopes.

and decrease hinged at $\mathrm{Kr}$ and $\mathrm{Xe}$; Xe has only one isotope less than those of Sn. To summarize the evolution of the ability to harbour neutrons, at the progress of $\mathrm{Z}$, reaches maxima at $\mathrm{Kr}$ and $\mathrm{Xe}$ and appear influenced by the magic neutron numbers 50 and 82 . For the sake of the ideas suggested here it is clear that the above-mentioned ability must extend above the $\beta$-stability valley into the region of the primary neutron-rich fragments of the nuclear fissions (Figure 1, Figure 5). The second consideration concerns the accurate experimental demonstration that, in the asymmetric fission of a long actinide chain from Th to Fm, the most probable charge of the heavy fragment is fixed at $Z=54$, the Xe charge [16]. Moreover in the $\beta$-delayed fission of the neutron poor ${ }^{180} \mathrm{Tl}$ nucleus the light 
fragment charge is $\mathrm{Z}=36$, the $\mathrm{Kr}$ charge, ruling out any possible dominance of the Xe charge of the heavy fragment. The proton numbers 36 and 54 appear hence somehow "magic" in spite of related shells absence in the shell model. In the long chain of actinide fission the $\mathrm{Z}=54$ charge of the heavy fragment automatically fixes the charge of the light fragment [16], because of the proton number conservation in nuclear fission. Then ${ }_{38} \mathrm{Sr}$ appears less magic than ${ }_{54} \mathrm{Xe}$ since its top stability (Figure 3 and Figure 5) is entirely due to the neutron magic number 50. The choice of Sr, to complement the most probable Sr-Xe couple of Figure 4, dictated by the Xe charge, appears in addition backed up by the top $\mathrm{BE}$ of the $\mathrm{Sr}$ isotope (Figure 3, Figure 5). An unknown physical situation helps the choice of the $Z_{\mathrm{L}}$ fragment, of the $\mathrm{Z}_{\mathrm{L}}-\mathrm{Z}_{\mathrm{H}}$ couples, in all of the low energy fissions of the actinides (Figure 3, Figure 6). Reasoning on the manifestation of fragment shells in nuclei undergoing fission the existence of a "shell" at $Z=54$ was suggested, [14] Figure 7. The same figure, elaborated from a study of nuclear fission

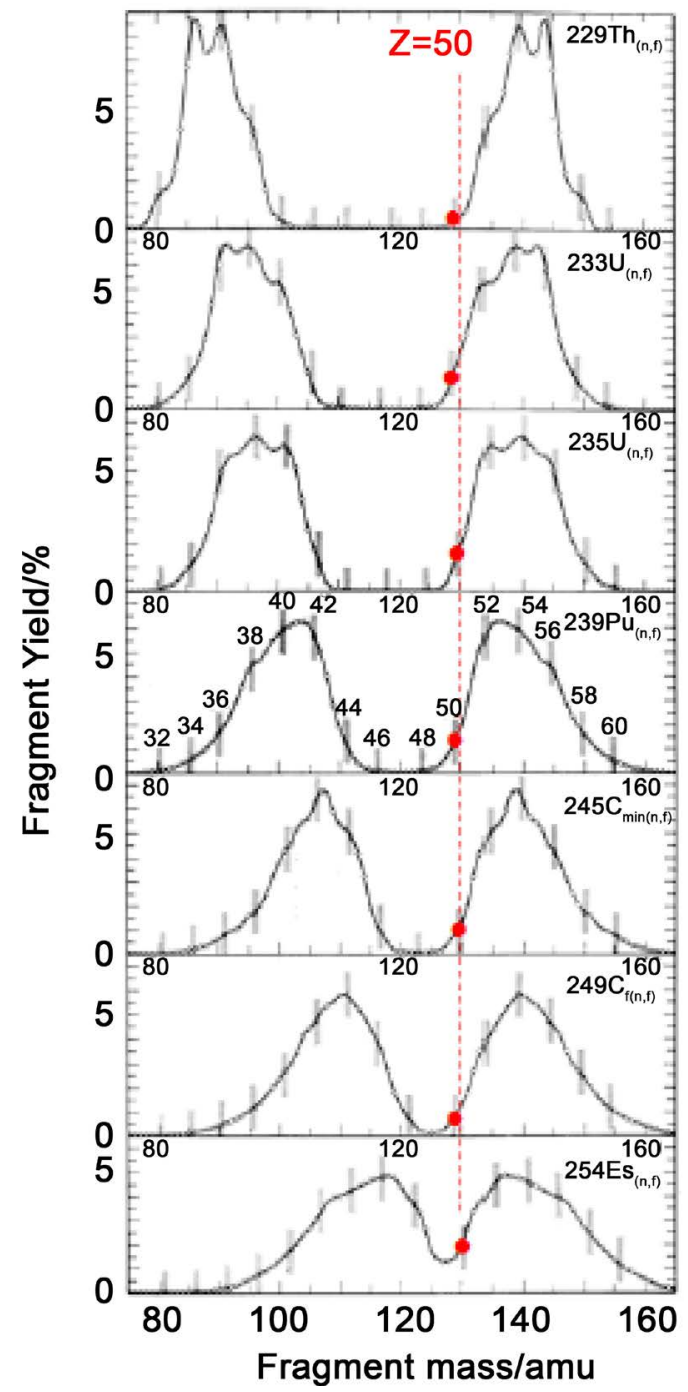

Figure 6. Asymmetric fission in thermal neutron fission from $\mathrm{Th}_{(\mathrm{n}, \mathrm{f})}$ to $\mathrm{Es}_{(\mathrm{n}, \mathrm{f})}$. Adaptation [10] of Figure 1 [22]. 


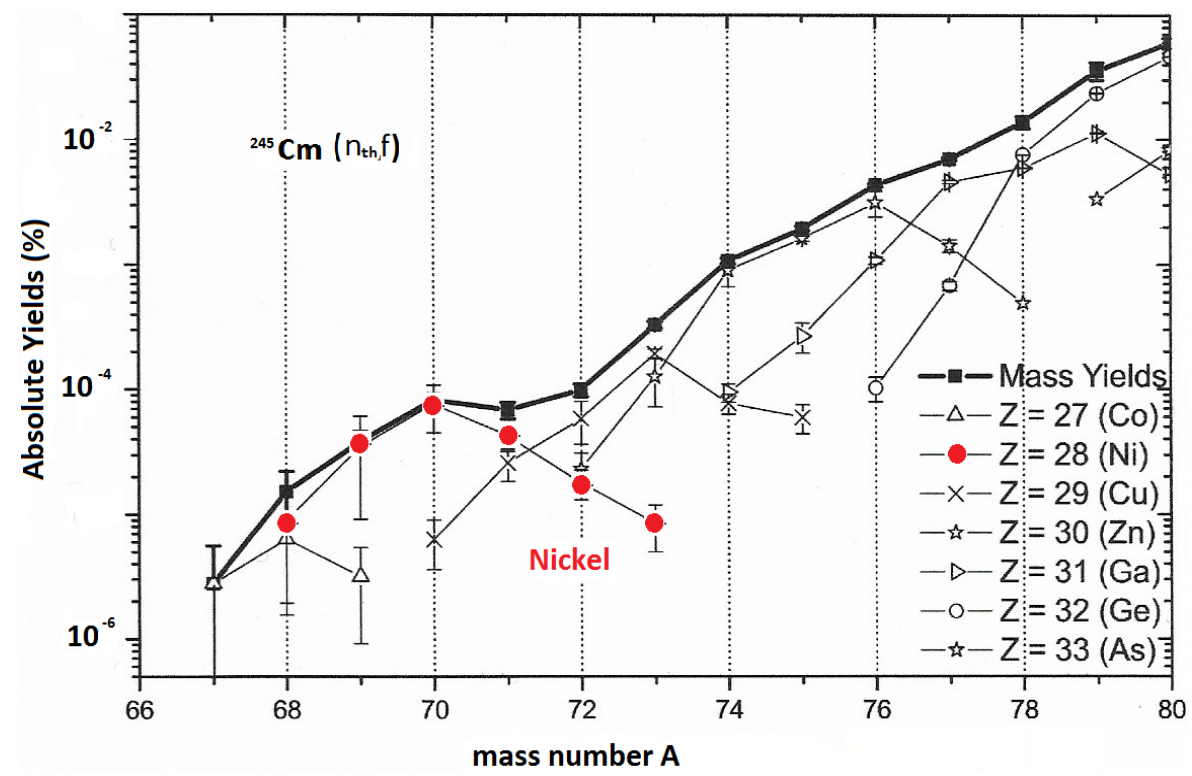

Figure 7. Proton rich isotopes of low $\mathrm{Z}$ from the superasymmetric fission of ${ }^{245} \mathrm{Cm}_{(\mathrm{nth}, \mathrm{f})}$. Adaptation [10] of Figure 3 [15].

down to ${ }_{83}$ Tl, Itkis et al. [21], may well justify a "shell" at $\mathrm{Z}=36$. The importance attributed here to the proton numbers 36 and 54 stems from the $\mathrm{Kr}$ and Xe features stressed above coupled with the experimental data. The physics behind the present idea awaits investigation. It appears hidden in the nature of the $\mathrm{Z}$ number.

The apparent slay down of the block of isotopes, encompassed between the $50 \mathrm{~N}$ and the $82 \mathrm{~N}$ arrays (Figure 1), caused by the NE lowering of the $50 \mathrm{~N}$ isoton array, if correct [19], has an interesting outcome. It offers a frame for the interpretation of the post fission evaporation of neutrons, higher for the $Z_{\mathrm{L}}$ compared to the $Z_{\mathrm{H}}$ fragments; a tenet in actinide fission. This is because the $50 \mathrm{~N}$ array interacts more extensively with the $Z_{\mathrm{L}}$ fragments than the $82 \mathrm{~N}$ array can do with the $\mathrm{Z}_{\mathrm{H}}$ fragments (Figure 4, Figure 6).

Figure 6 stresses that, for all thermal neutron actinide fissions, the yields of the heavy mass fragment groups start rising at $A_{H}=130 \mathrm{amu}$, corresponding to the magic proton charge $\mathrm{Z}=50$. At ${ }^{100} \mathrm{Sn}^{50}$ the suggested reduction of the element's ability to harbour the EN vanishes, because the EN zeroes, and the element $\mathrm{Sn}$ rises its ability to harbour the NE. See the sharp rise, from $\mathrm{Cd}$ to $\mathrm{Sn}$, of the NE accommodation ability (Figure 1). This observation from one side gives further credit to the element's isotopes layout interpretation [19] and for the other side offers a hint for the interpretation of the 50 proton $\mathrm{Z}$ charge function in the Figure 6.

The importance of the 28 magic proton number in nuclear fission (Figure 3) is evident by the yield shoulder marked by ${ }^{70} \mathrm{Ni}$ isotope (Figure 7). At variance with Figure 4, the fission fragments yield is extended dawn to $10^{-6} \%$.

Few additional comments. Figure 1 highlights, besides the double magic ${ }^{78} \mathrm{Ni}^{28}$ $\left(\mathrm{Ni}_{22}\right)$ and ${ }^{132} \mathrm{Sn}^{82}\left(\mathrm{Sn}_{32}\right)$ other interesting isotopes (Figure 1 legend). With the 
help of the NE numbers, it is easy to spot these isotopes in Figure 3 and in Figure 5. The ${ }^{70} \mathrm{Ni}$ isotope is important because it marks the yield shoulder in the super asymmetric fission of ${ }^{245} \mathrm{Cm}_{(\mathrm{n}, \mathrm{f})}$ and of others actinides, Figure 7 reproduced with permission [10]. The post neutron evaporation fragments and their $\beta$-decay to stable isotopes are an example of the ${ }^{235} \mathrm{U}_{(\mathrm{n}, \mathrm{f})}$ asymmetric fission [20]. The isotopic couples $\mathrm{Xe}-\mathrm{Sr}, \mathrm{Te}-\mathrm{Zr}$ and $\mathrm{Ba}-\mathrm{Kr}$ represent the pre neutron evaporation fragments of the three most probable charge yield in the electromagnetic induced fission of ${ }^{234} \mathrm{U}$ shown in Figure 4 [11]. With the exception of the BE of $\mathrm{Ni}_{22}$, lying at the border of the neutron drip line, all other neutron rich isotopes appear removed from that line (Figure 3) [19].

\section{Concluding Remarks}

The element's ability to harbour the NE (Figure 1 ) is central in nuclear fission. $\mathrm{Xe}$ and $\mathrm{Kr}$ proton charges rule the actinide and the ${ }^{180} \mathrm{Tl} \beta$-delayed fission respectively, however all element's proton numbers are important. In actinide fission the choice of $\mathrm{Sr}$, in the most probable Sr-Xe caple, is not casual (Figure 3). An unknown physical determinant complement the fragment's charges setting by the Xe charge. The phenomenon is general assisting all the fragmentations in actinide fission and the charge-mass staggering effect.

\section{Acknowledgements}

The author thanks Maurizio Zanardini for the graphical work.

\section{References}

[1] Bohr, N. and Wheeler, J.A. (1939) The Mechanism of the Nuclear Fission. Physical Review, 56, 426.

[2] Meitner, L. and Frisch, O.R. (1939) Products of the Fission of the Uranium Nucleus. Nature, 143, 471-472. https://doi.org/10.1038/143471a0

[3] Hamamoto, I. and Mottelson, B.R. (2012) Shape Deformation in Atomic Nuclei. Scholarpedia, 7, 10693. https://doi.org/10.4249/scholarpedia.10693

[4] Frankel, S. and Metropolis, N. (1947) Calculations in the Liquid-Drop Model of Fission. Physical Review, 72, 914. https://doi.org/10.1103/PhysRev.72.914

[5] Swiateski, W.J. (1955) Systematics of Spontaneous Fission Half-Lives. Physical Review, 100, 937. https://doi.org/10.1103/PhysRev.100.937

[6] Goeppter Mayer, M. (1963) The Shell Model. Nobel Lecture. http://www.nobelprize.org/nobel_prizes/physics/laureates/1963/mayer/lecture.html

[7] Jansen, J.H.D. (1963) Glimpses at the History of the Nuclear Structure Theory. Nobel Lecture. http://www.nobelprize.org/nobel_prizes/physics/laureates/1963/jensen/lecture.html

[8] Strutinsky, W.M. (1967) Shell Effects in Nuclear Masses and Deformations Energies. Nuclear Physics A, 95, 420-444. https://doi.org/10.1016/0375-9474(67)90510-6

[9] Strutinsky, W.M. (1968) Shells in Deformed Nuclei. Nuclear Physics A, 122, 1-33. https://doi.org/10.1016/0375-9474(68)90699-4

[10] Gönnenwein, F. (2014) Neutron-Induced Fission. The Ecole Joliot Curie, Frejus. http://ejc2014.sciencesconf.org/conference/ejc2014/pages/goennenwein2.pdf 
[11] Möller, P., Madland, D.G. and Ivamoto, A. (2001) Nuclear Fission Modes and Fragment Mass Asymmetries in Five-Dimensional Deformation Space. Nature, 409, 785-790. https://doi.org/10.1038/35057204

[12] Schmidt, K.-H., Benlliure, J. and Junghans, A.R. (2001) Fission of Nuclei Far from Stability. Nuclear Physics A, 693, 169-189. https://doi.org/10.1016/S0375-9474(01)00648-0

[13] Schmidt, K.-H. and Jurado, B. (2012) Global View on Fission Observables-New Insights and New Puzzles. Physics Procidia, 31, 147-157. https://doi.org/10.1016/j.phpro.2012.04.020

[14] Schmidt, K.-H. and Jurado, B. (2014) General View on the Progress in Nuclear Fission: A Review. HAL Id: in2p3-01314814. http://hal.in2p3.fr/in2p3-01314814

[15] Rochman, D., Tsekhanovich, I., Gonnenwein, F., Sokolov, V., Storrer, F., Simpson, G. and Serot, O. (2004) Super-Asymmetric Fission in the ${ }^{245} \mathrm{Cm}\left(\mathrm{n}_{\mathrm{th}}, \mathrm{f}\right)$ Reaction at the Lohengrin Fission-Fragment Mass Separator. Nuclear Physics A, 735, 3-20. https://doi.org/10.1016/j.nuclphysa.2004.01.121

[16] Schmidt, K.-H. and Jurado, B. (2010) General Model Description of Fission Observables. http://www.cenbg.in2p3.fr/IMG/pdf_final_efnudat_report2.pdf

[17] Elseviers, J., et al. (2013) $\beta$-Delayed Fission of ${ }^{180}$ Tl. Physical Review C, 88, 044321. https://doi.org/10.1103/PhysRevC.88.044321

[18] Audi, G., Wapstra, A.H. and Thibault, C. (2003) The $\mathrm{A}_{\mathrm{ME}} 2003$ Atomic Mass Evaluation: (II). Tables, Graphs and References. Nuclear Physics A, 729, 337-676.

[19] Menegus, F. (2016) The Representation of the Chemical Element's Isotopes by the Neutron Excess Content. World Journal of Nuclear Science and Technology, 6, 147-152. https://doi.org/10.4236/wjnst.2016.63016

[20] Cook, N.D. (2010) Models of the Atomic Nucleus. 2nd Edition, Springer-Verlag, Berlin Heidelberg, 163.

[21] Itkis, M.G., Okolovich, V.N., et al. (1985) Asymmetric Fission of the Pre-Actinide Nuclei. Zeitschrift für Physik A Atoms and Nuclei, 320, 433-441.

[22] Unik, J.P., Gindler, J.E., Glendenin, J.E., et al. (1974) Proc. Phis. and Chem. of Fission, Vol II, IEA, Vienna. 